\title{
Ultraconserved Long Non-Coding RNA Uc.112 is Abnormally Expressed in Childhood Acute Lymphoblastic Leukemia Subtypes
}

\author{
Pablo Ferreira das Chagas ${ }^{1,2}$, Graziella Ribeiro de Sousa ${ }^{2}$, Márcio Hideki Kodama², Carlos \\ Alberto Oliveira de Biagi Junior'2 José Andres Yunes ${ }^{3}$, Silvia Regina Brandalise ${ }^{3}$, George Adrian \\ Calin ${ }^{4}$, Luiz Gonzaga Tone ${ }^{2}$, Carlos Alberto Scrideli ${ }^{2}$ and Jaqueline Carvalho de Oliveira ${ }^{1,5 *}$ \\ 1 Federal University of Alfenas, Alfenas, Minas Gerais, Brazil \\ 2 Faculty of Medicine of Ribeirão Preto, University of São Paulo, Ribeirão Preto, São Paulo, Brazil \\ 3 Boldrini Institute, Campinas, São Paulo, Brazil \\ 4 MD Anderson Cancer Center, Houston, TX, United States \\ 5 Federal University of Parana, Curitiba, Parana, Brazil \\ pablochagas@usp.br (P.F.C.); graziellasousa@usp.br (G.R.S.); marciohkodama@usp.br (M.H.K.); biagi@usp.br \\ (C.A.O.B.J.); andres@boldrini.org.br (J.A.Y.); silvia@boldrini.org.br (S.R.B.); gcalin@mdanderson.org (G.A.C.); \\ lgtone@fmrp.usp.br (L.G.T.); scrideli@fmrp.usp.br (C.A.S.) \\ * Correspondence: jaqueline.carvalho@ufpr.br; Tel.: +55-41-3361-1725
}

\begin{abstract}
Long non-coding RNA (lncRNA) aberrant expression have been found in several types of cancer, including acute lymphoblastic leukemia (ALL), but lncRNA mapped in transcribed ultraconserved regions (T-UCRs) are little explored. The T-UCRs uc.112, uc.122, uc.160 and uc.262 were evaluated in pediatric ALL and uc.112 expression was higher in T-ALL compared to patients with B-ALL and in patients with hyperdiploid karyotype. These findings suggest a potential role of this uc.112 in pediatric ALL and emphasize the need for further investigation of T-UCR in pediatric ALL.
\end{abstract}

Keywords: T-UCR; pediatric acute lymphoblastic leukemia; uc.112; T-ALL; hyperdiploidy.

\section{Introduction}

Acute lymphoblastic leukemia (ALL) represents the most common childhood malignant neoplasia. Despite progress in current treatment, still almost $20 \%$ of affected children relapse and the causes are still unknown [1]. Considering that ALL is a heterogeneous disease, new efforts aim to understand the mean features of each subtype, including differences between T-ALL and precursor B-ALL.

Recently, long non-coding RNAs (lncRNAs) expression patterns have been highly informative in several types of cancer, including ALL $[2,3]$. Curiously, many lncRNAs are poorly conserved among different species when compared with protein-coding genes [4]. However, this lack of sequence conservation is not true for lncRNA mapped at ultraconserved regions.

Ultraconserved regions (UCR) are 481 segments, longer than $200 \mathrm{nt}$, initially identified as result of bioinformatic comparisons between human, mouse and rat genome: these regions are widely distributed, overlap exonic, intronic and intergenic regions [5]. Additionally, the majority of these regions are expressed in normal tissues, so called transcribed UCRs (T-UCRs) [6].

The initial evidence of T-UCR association with cancer was due to distinct expression signatures in adult chronic lymphocytic leukemia, colorectal and hepatocellular carcinoma [6]. Since then, many studies have shown that altered T-UCR expression is associated with several human cancers [7]. But, in patients with ALL, these regions have not been investigated. 
In the present study, we aimed to evaluate the expression level of uc.112, uc.122, uc.160 and uc.262, intergenic T-UCR differentially expressed in cancer patients [6] in children with T-ALL and precursor B-ALL.

\section{Results}

Higher expression levels of uc.112 were found in patients with T-ALL compared to patients with B-ALL ( $\log 2$ foldchange 4.08, $\mathrm{P}=0.003$ ) (Figure 1A). Evaluating uc.112 expression in non-tumor B and $\mathrm{T}$ precursor from public data [8], no significant difference was found between $\mathrm{B}$ versus T-cell precursor (log2foldchange $0.82, \mathrm{P}=0.49$, Salmon analysis; log2foldchange $0.88, \mathrm{P}=0.46$, Kallisto analysis).

Additionally, among B-ALL patients, uc.112 were also found increased in patients with hyperdiploidy ( $\mathrm{P}=0.0003$ ) (Figure $1 \mathrm{~B})$. Event-free survival was not significantly different according to uc.112 expression values (Figure 2). Uc.122, uc.160 and uc.262 were not associated with analyzed variables, including survival analysis.

Using bioinformatics approaches [9], we evaluated potential regulatory elements associated with uc.112 genomic position. Uc.112 region was bound with the transcription factors NFIC, ATF2, MAZ, SP1, TBL1XR1, BCLAF1, PAX5, POU2F2, IRF4, RUNX3, EBF1, BATF, BCLA11 only in GM12878 cell line (Figure 2). GM12878 is a B-lymphoblastoid cell line established by Epstein-Barr Virus transformation of peripheral blood mononuclear. The factor SPI1 was also found in GM12878 and in K562 cells, isolated from chronic myelogenous leukemia.

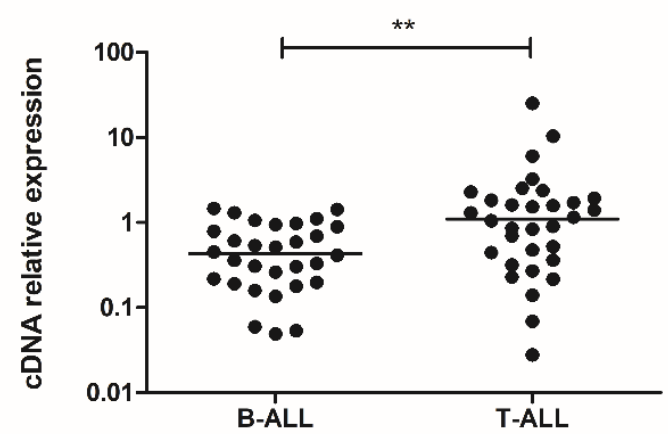

(a)

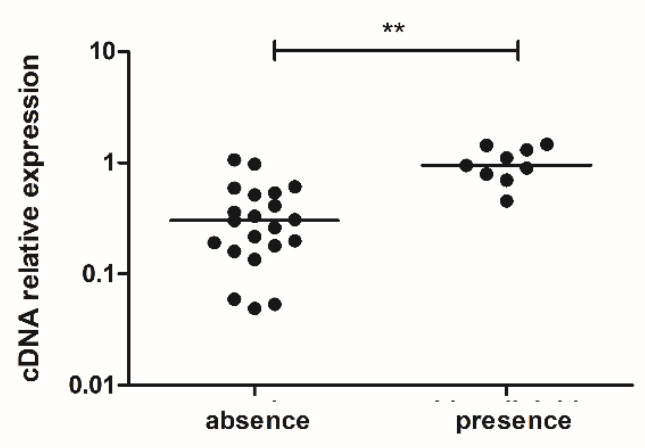

(b)

Figure 1. Uc.112 analyses in pediatric acute lymphoblastic leukemia (ALL). (a) Uc.112 expression in patients with B-cell phenotype (left) or T-cell phenotype (right). (b) Uc.112 expression in B-ALL patients with absence of hyperdiploidy karyotype (left) and presence of hyperdiploid karyotype (right). ${ }^{* *} \mathrm{P}<0.001$, Mann-Whitney test. 


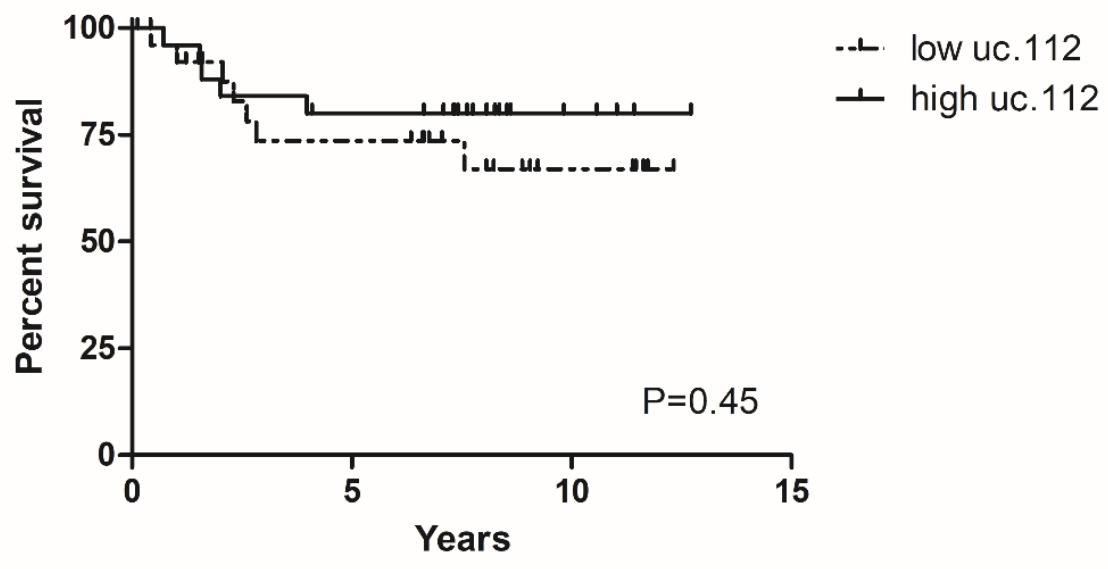

Figure 2. Event-free survival (EFS) determined by Kaplan-Meier plots and the log-rank test according to higher or lower than median gene expression values of uc.112.

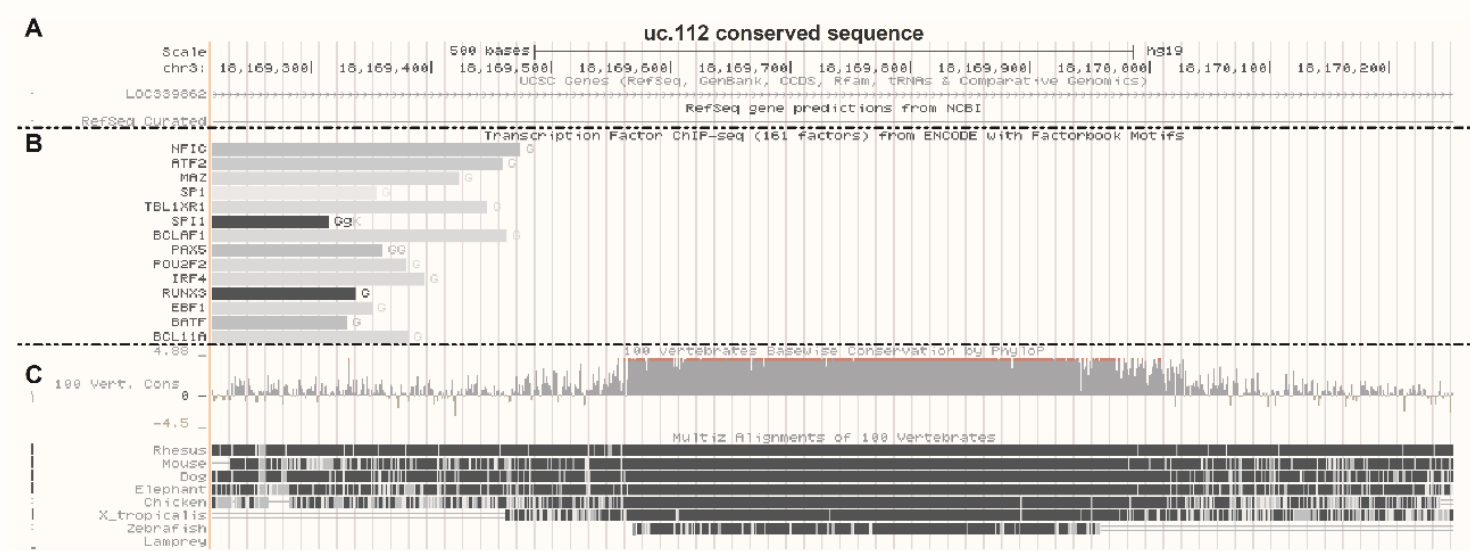

Figure 3. UCSC Genome Browser histogram showing uc.112 locus. Accessing the position chr3:1816956418169909, GRCh37/hg19 Assembly, and visualized with zoom out of 3 times. (A) Chromosome localization. (B) Transcription factor immunoprecipitated according ENCODE data (C) Conservation information.

\section{Discussion}

Since the initial evidence of T-UCR association with cancer [6] many others have emphasized TUCR deregulation in several tumor types. For example, Scaruffi et al. showed that T-UCRs expression is associated with outcome in high-risk neuroblastoma (NB) [10]. Likewise, uc.347, uc.350, uc.279, uc.379, uc.460, uc.446 e uc.364 were identified upregulated in NB with MYCN amplification [11]. In addition, after retinoic acid treatment the levels of uc.300a expression were linked with proliferation, invasion and the inhibition of differentiation of NB cell lines [12].

In colorectal cancer, uc.73 and uc.388 were decreased whereas uc.73 indicated a positive correlation with overall survival [13]. By the other hand, in human carcinoma hepatocellular, uc.338 is upregulated in compared with noncancerous adjacent tissues [14]. Further, low expression of uc. 306 was associated with shorter overall survival in hepatitis B (HBV)-related hepatocarcinoma [15].

In Barrett-related adenocarcinoma also was found upregulation of uc.58, uc.202, uc.207, and uc.223 and downregulation of uc.214 [16] and, in pancreatic adenocarcinoma, high levels of uc.190, uc.233 e uc.270 were identified [17]. Overexpression of uc.8 results increased expression of metalloprotease 9, increasing the invasive potential of bladder cancer cells [18]. 
In prostate cancer, Hudson et al performed analysis using a custom microarray with 962 probe sets and observed altered of uc.106, uc.477, uc.363 and uc.454 significantly deregulated [19], moreover, uc.63 promotes resistance to docetaxel through regulation of androgen receptor signaling by interacting with miR-130b [20]. Uc.63 expression is also associated with bad outcome in luminal A subtype of breast cancer patients [21]. And expression of uc.38 also is downregulated in breast cancer cells inducting apoptosis and features associated with progression of this tumor [22].

In hematological disease only CLL has description about T-UCRs deregulation. Calin et al, with the pioneer description of T-UCR including CLL patients [5], Wojcik et al, evidencing DNA sequence variations in miRNAs and ultraconserved genes in colorectal cancer and CLL [23] and, recently, description of the prognostic value of uc.70 in CLL patients [24].

The intergenic T-UCRs evaluated in present study, in ALL patients, according to Calin et al. [6], were deregulated in some type of cancer. Uc.122, uc.160, uc.262 were up regulated in chronic lymphocytic leukemia (CLL) and uc.112 were high expressed in colorectal cancer. Despite description of some T-UCR pattern expression in tumor versus normal counterpart, few descriptions focus on differences in subtypes in a specific disease.

Herein, high uc.112 expression was found in patients with T-ALL and in patients with hyperdiploid karyotype of more than 50 chromosomes. ENCODE data show that all transcription factors found in uc.112 region were detected in lymphocytes and may provide some clues for T-ALL versus B-ALL difference expression.

Differentiation of hematopoietic stem cells into B lymphocytes requires the concerted action of specific transcription factors, such as PAX5, IRF4, EBF1, RUNX1 and others, some of these bounding in uc.112 genomic region. For example, PAX5, IRF4, EBF1 and TBL1XR1 are found associated to uc.112 region and they are usually found genetically altered in a large portion of patients with B-ALL [25-27]. Additionally, RUNX3, downstream effector of NOTCH pathway, and KLF4, inactivated in children with T-ALL [28], were reported to bound to uc.112 on CHIPseq data.

Uc.122 was previously detected high expressed in CLL and associated with better outcome in neuroblastoma [10]. Uc.160 was detected previously increased in CLL [6] and, recently, was found down regulated in gastric cancer [29]. Additionally, uc.262 as one of the top UCRs differentially expressed in granulocyte linages expression when it was evaluated differentiation to the erythrocyte, megakaryocyte, monocyte and granulocyte lineages [30]. But, in ALL, the relevance of uc.122, uc.160 and uc.262 were not found.

In conclusion, uc.112 was found high expressed in T-ALL compared to patients with B-ALL and, among B-ALL, uc.112 was found increased in patients with hyperdiploid karyotype. To our knowledge, this is the first study to analyze some T-UCRs expression in ALL patients and our results emphasize the need for further investigation of T-UCR in pediatric ALL, mainly in specific subtypes.

\section{Materials and Methods}

\subsection{Patients}

Bone marrow samples were obtained at diagnosis from patients with T-ALL $(n=32)$ and common-ALL/pre-B ALL ( $\mathrm{n}=30)$, treated according to the Brazilian Childhood Leukemia Treatment protocol [31]. Among patients with B-ALL samples, seven patients presented $t(12 ; 21)$ and nine present hyperdiploid. Unfavorable events (death due to any cause or relapse) occurred in 17 patients. Additional biological and clinincal features are described in Table S1. The study was approved by the National Research Ethics Committee (CONEP, No. 7329/2009) and samples were collected after written informed consent.

\subsection{RNA extraction, cDNA synthesis and quantitative real-time PCR ( $q R T-P C R$ )}

Mononucleated cells were separated by a Ficoll-Paque centrifugation gradient and total cellular RNA was extracted using Trizol Reagent (Invitrogen, Carlsbad, CA, USA) and checked for RNA integrity by an Agilent Bioanalyzer 2100 (Agilent technologies, Santa Clara, CA, US). 800 ng of total RNA, with RIN above 7 and treated with DNAse $(0,4 \mathrm{U})$, was retrotranscribed with random primers and Superscript III (Applied Biosystems, Foster City, CA), and RT-qPCR was performed using SYBR 
Green dye staining. Each $20 \mu \mathrm{l}$ reaction contained $0.5 \mu \mathrm{l}$ cDNA, 1x Power SYBR Green PCR Master Mix (Applied Biosystems) and $200 \mathrm{nM}$ each primer. The primers sequence used are described in Table S2 and, amplification efficiency of each primer was $95-105 \%$.

T-UCR levels were measured using the ABI 7500 Real Time PCR System (PE Applied Biosystems). The relative expression was calculated using the 2 - $\mathrm{ACT}$ method with two internal controls, GUS and GAPDH and expression in ReH cell line was used as calibrator in all reaction. qRTPCR was performed in triplicate and maximum standard deviation of $<0.5$ was accepted. A blank control was run in parallel within each experiment.

\subsection{Statistical analysis}

A pilot study was performed with 18 patients samples for evaluate an effective expression of 6 T-UCRs region (uc.112, uc.122, uc.160, uc.252, uc.262, uc.316) but uc.252 and uc.316 expression were detected in less than $45 \%$ of the analyzed samples and were removed from the future analysis. Small variation on GUS and GAPDH endogenous expression across different expression was also checked. After these preliminary analyses, $30 \mathrm{~B}$-ALL and 32 T-LLA sample were evaluated.

The association between the analyzed variables (age, white blood cell count at diagnosis, immunophenotype, hyperdiploid karyotype and $t(12 ; 21)$ presence) and T-UCRs expression levels was determined by the Mann-Whitney test. $\mathrm{Ct}$ values of endogenous had no difference $(\mathrm{P}>0.05)$ among variables analyzed (Figure S1).

Patients were stratified in values below and above the median for survival analyses. The Kaplan Meier curves and log rank test were used to estimate event-free survival (5-year EFS), which was calculated from the date of complete continuous remission to the last follow-up or unfavorable event (induction failure, relapse and/or death).

\subsection{Bioinformatics analysis}

Aiming to evaluate uc.112 expression in non-tumor B and T blast cells, we extracted RNAseq data from Casero et al., 2015 [8] and evaluated uc.112 expression in fully B cell committed progenitors (CD34+CD38+CD19+) from bone marrow and fully $\mathrm{T}$ cell committed populations (CD4+CD8+) from thymus.

First, we download the raw sequence files that are available at the NCBI Gene Expression Omnibus website with accession code GSE69239. The FastQC [32], MultiQC [33] and Trim Galore [34] softwares were used to analyze the quality control on raw sequence data and adapter trimming. Then Salmon [35] and Kallisto [36] were used to quantify the abundances of transcripts. These methods are based on the idea of pseudoalignment determining the compatibility of reads with targets, without the need for alignment. The tximport [37] package was used to read the previously generated quantification files thus generating the counting table that was used in differential expression analysis from the DESeq2 [38] package.

With "The Encyclopedia of DNA Elements (ENCODE)" data available at University of California, Santa Cruz (UCSC) platform (http://genome.ucsc.edu/), regulatory elements associated to genomic position of uc.112 were evaluated.

Accessing UCSC Genome Browser GRCh37/hg19 Assembly, the position chr3:1816956418169909 were visualized with zoom out of $3 x$ and include information of transcription factors by combining chromatin immunoprecipitation assays with sequencing (ChIP-seq). UCSC contained transcription factor ChIP-seq evaluated of 161 factors in 91 cell types from ENCODE.

Supplementary Materials: The following are available online at www.mdpi.com/xxx/s1, Figure S1: Ct values for endogenous control in "B-ALL vs T-ALL" (A.B) and "hiperdiploid vs negative" (C.D) samples. P > 0.05, Mann-Whitney test., Table S1: Expression data and patient features. Table S2: Primers sequences used in quantitative real time PCR.

Author Contributions: P.F.C., G.A.C., C.A.S. and J.C.O. designed the experiments; P.F.C., G.R.S., M.H.K., M.I. and J.C.O conceived the experiments and analyzed the data; J.A.Y., S.R.B., L.G.T. and C.A.S. contributed to patients recruitment, sample and clinical data collection, P.F.C. and J.C.O. drafted the paper. All authors critically reviewed the article. 
Funding: This collaborative study was supported by the Public Research Agencies: Conselho Nacional de Desenvolvimento Científico e Tecnológico (CNPq), grants no. 442583/2014-1, CAPES, FAPEMIG, Fundação de Amparo a Pesquisa do Estado de Sao Paulo (FAPESP), and FAEPA (Brazil).

Acknowledgments: Leilane Sales de Oliveira and Rafael Fernandes Martins for technical assistance..

Conflicts of Interest: The authors declare no conflict of interest.

\section{References}

1. Pui, C.H.; Yang, J.J.; Hunger, S.P.; Pieters, R.; Schrappe, M., Biondi, A., Escherich, G. Childhood acute lymphoblastic leukemia: progress through collaboration. J Clin Oncol 2015; 33(27), 2938-2948, DOI: 10.1200/JCO.2014.59.1636.

2. Lin, C.; Yang, L. Long Noncoding RNA in Cancer: Wiring Signaling Circuitry. Trends Cell Biol 2018, 28(4), 287-301, DOI: 10.1016/j.tcb.2017.11.008.

3. Gioia, R.; Drouin, S.; Ouimet, M.; Caron, M.; St-Onge, P.; Richer, C.; Sinnett, D. LncRNAs downregulated in childhood acute lymphoblastic leukemia modulate apoptosis, cell migration, and DNA damage response. Oncotarget 2017, 8(46), 80645-80650. DOI: 10.18632/oncotarget.20817.

4. Johnsson, P.; Lipovich, L.; Grandér, D.; Morris, K.V. Evolutionary conservation of long non-coding RNAs; sequence, structure, function. Biochim Biophys Acta 2014, 1840(3), 1063-1071, DOI: 10.1016/j.bbagen.2013.10.035.

5. Bejerano, G.; Pheasant, M.; Makunin, I.; Stephen, S.; Kent, W.J.; Mattick, J.S.; Haussler, D. Ultraconserved elements in the human genome. Science 2004, 304(5675), 1321-1325, DOI: 10.1126/science.1098119.

6. Calin, G.A.; Liu, C.G.; Ferracin, M.; Hyslop, T.; Spizzo, R.; Sevignani, C.; Fabbri, M.; Cimmino, A.; Lee, E.J.; Wojcik, S.E.; Shimizu, M.; Tili, E.; Rossi, S.; Taccioli, C.; Pichiorri, F.; Liu, X.; Zupo, S.; Herlea, V.; Gramantieri, L.; Lanza, G.; Alder, H.; Rassenti, L.; Volinia, S.; Schmittgen, T.D.; Kipps, T.J.; Negrini, M.; Croce, C.M. Ultraconserved regions encoding ncRNAs are altered in human leukemias and carcinomas. Cancer cell 2007, 12(3), 215-229, DOI: 10.1016/j.ccr.2007.07.027.

7. Fabris, L., Calin, G.A. Understanding the Genomic Ultraconservations: T-UCRs and Cancer. Int Rev Cell Mol Biol 2017, 333: 159-172. DOI: 10.1016/bs.ircmb.2017.04.004 .

8. Casero, D.; Sandoval, S.; Seet, C.S.; Scholes, J.; Zhu, Y.; Ha, V.L.; Luong, A.; Parekh, C.; Crooks, G.M. Long non-coding RNA profiling of human lymphoid progenitor cells reveals transcriptional divergence of B cell and T cell lineages. Nat Immunol 2015, 16(12), 1282-1291, DOI: 10.1038/ni.3299.

9. Rosenbloom, K.R.; Dreszer, T.R.; Long, J.C.; Malladi, V.S.; Sloan, C.A.; Raney, B.J.; Cline, M.S.; Karolchik, D.; Barber, G.P.; Clawson, H.; Diekhans, M.; Fujita, P.A.; Goldman, M.; Gravell, R.C.; Harte, R.A.; Hinrichs, A.S.; Kirkup, V.M.; Kuhn, R.M.; Learned, K.; Maddren, M.; Meyer, L.R.; Pohl, A.; Rhead, B.; Wong, M.C.; Zweig, A.S.; Haussler, D.; Kent, W.J. ENCODE whole-genome data in the UCSC Genome Browser: update 2012. Nucleic Acids Res 2012, 40: D912-D917, DOI: 10.1093/nar/gkr1012.

10. Scaruffi, P; Stigliani, S.; Moretti, S.; Coco, S.; De Vecchi, C.; Valdora, F.; Garaventa, A.; Bonassi, S.; Tonini, G.P. Transcribed-Ultra Conserved Region expression is associated with outcome in high-risk neuroblastoma. BMC Cancer 2009, 9: 441. doi: 10.1186/1471-2407-9-441.

11. Mestdagh, P.; Fredlund, E.; Pattyn, F.; Rihani, A.; Van Maerken, T.; Vermeulen, J.; Kumps, C.; Menten, B.; De Preter, K.; Schramm, A.; Schulte, J.; Noguera, R.; Schleiermacher, G.; Janoueix-Lerosey, I.; Laureys, G.; Powel, R.; Nittner, D.; Marine, J.C.; Ringnér, M.; Speleman, F.; Vandesompele J. An integrative genomics screen uncovers ncRNA T-UCR functions in neuroblastoma tumours. Oncogene 2010, 29(24): 3583-3592. DOI: 10.1038/onc.2010.106.

12. Watters, K.M.; Bryan, K.; Foley, N.H.; Meehan, M.; Stallings, R.L. Expressional alterations in functional ultra-conserved non-coding RNAs in response to all-trans retinoic acid--induced differentiation in neuroblastoma cells. BMC Cancer 2013, 13:184. DOI: 10.1186/1471-2407-13-184.

13. Sana, J.; Hankeova, S.; Svoboda, M.; Kiss, I.; Vyzula, R.; Slaby O. Expression levels of transcribed ultraconserved regions uc.73 and uc.388 are altered in colorectal cancer. Oncology 2012, 82(2): 114-118. DOI: $10.1159 / 000336479$.

14. Braconi, C.; Valeri, N.; Kogure, T.; Gasparini, P.; Huang, N.; Nuovo, G.J.; Terracciano, L.; Croce, C.M.; Patel, T. Expression and functional role of a transcribed noncoding RNA with an ultraconserved element in hepatocellular carcinoma. Proc Natl Acad Sci U S A 2011, 108(2): 786-91. DOI: 10.1073/pnas.1011098108. 
15. Luo, H.L.; Chen, J.; Luo, T.; Wu, F.X.; Liu, J.J.; Wang, H.F.; Chen, M.; Li, L.Q.; Li, H. Downregulation of Macrophage-Derived T-UCR uc.306 Associates with Poor Prognosis in Hepatocellular Carcinoma. Cell Physiol Biochem 2017, 42(4): 1526-1539. DOI: 10.1159/000479269.

16. Fassan, M.; Dall'Olmo, L.; Galasso, M.; Braconi, C.; Pizzi, M.; Realdon, S.; Volinia, S.; Valeri, N.; Gasparini, P.; Baffa, R.; Souza, R.F.; Vicentini, C.; D'Angelo, E.; Bornschein, J.; Nuovo, G.; Zaninotto, G.; Croce, C.M.; Rugge, M. Transcribed ultraconserved noncoding RNAs (T-UCR) are involved in Barrett's esophagus carcinogenesis. Oncotarget 2014, 5(16): 7162-71. DOI: 10.18632/oncotarget.2249

17. Jiang, J.; Azevedo-Pouly, A.C.; Redis, R.S.; Lee, E.J.; Gusev, Y.; Allard, D.; Sutaria, D.S.; Badawi, M.; Elgamal, O.A.; Lerner, M.R.; Brackett, D.J.; Calin, G.A.; Schmittgen, T.D. Globally increased ultraconserved noncoding RNA expression in pancreatic adenocarcinoma. Oncotarget 2016, 7(33): 53165-53177. DOI: 10.18632/oncotarget.10242.

18. Olivieri, M.; Ferro, M.; Terreri, S.; Durso, M.; Romanelli, A.; Avitabile, C.; De Cobelli, O.; Messere, A.; Bruzzese, D.; Vannini, I.; Marinelli, L.; Novellino, E.; Zhang, W.; Incoronato, M.; Ilardi, G.; Staibano, S.; Marra, L.; Franco, R.; Perdonà, S.; Terracciano, D.; Czerniak, B.; Liguori, G.L.; Colonna, V.; Fabbri, M.; Febbraio, F.; Calin, G.A.; Cimmino, A. Long non-coding RNA containing ultraconserved genomic region 8 promotes bladder cancer tumorigenesis. Oncotarget 2016, 7(15): 20636-20654. DOI: 10.18632/oncotarget.7833.

19. Hudson, R.S.; Yi, M.; Volfovsky, N.; Prueitt, R.L.; Esposito, D.; Volinia, S.; Liu, C.G.; Schetter, A.J.; Van Roosbroeck, K.; Stephens, R.M.; Calin, G.A.; Croce, C.M.; Ambs, S. Transcription signatures encoded by ultraconserved genomic regions in human prostate cancer. Mol Cancer 2013, 12:13. DOI: 10.1186/1476-459812-13.

20. Sekino, Y.; Sakamoto, N.; Goto, K.; Honma, R.; Shigematsu, Y.; Sentani, K.; Oue, N.; Teishima, J.; Matsubara, A.; Yasui, W. Transcribed ultraconserved region Uc.63+ promotes resistance to docetaxel through regulation of androgen receptor signaling in prostate cancer. Oncotarget 2017, 8(55): 94259-94270. DOI: 10.18632/oncotarget.21688.

21. Marini, A.; Lena, A.M.; Panatta, E.; Ivan, C.; Han, L.; Liang, H.; Annicchiarico-Petruzzelli, M.; Di Daniele, N.; Calin, G.A.; Candi, E.; Melino, G. Ultraconserved long non-coding RNA uc.63 in breast cancer. Oncotarget 2017, 8(22): 35669-35680. DOI: 10.18632/oncotarget.10572.

22. Zhang, L.X.; Xu, L.; Zhang, C.H.; Lu, Y.H.; Ji, T.H.; Ling, L.J. uc.38 induces breast cancer cell apoptosis via PBX1. Am J Cancer Res 2017, 7(12): 2438-2451.

23. Wojcik, S.E.; Rossi, S.; Shimizu, M.; Nicoloso, M.S.; Cimmino, A.; Alder, H.; Herlea, V.; Rassenti, L.Z.; Rai, K.R.; Kipps, T.J.; Keating, M.J.; Croce, C.M.; Calin, G.A. Non-codingRNA sequence variations in human chronic lymphocytic leukemia and colorectal cancer. Carcinogenesis 2010, 31(2): 208-215. DOI: 10.1093/carcin/bgp209.

24. Bomben, R.; Roisman, A.; D'Agaro, T.; Castellano, G.; Baumann, T.; Delgado, J.; López-Guillermo, A.; Zucchetto, A.; Dal-Bo, M.; Bravin, V.; Slavutsky, I.; Vlasova, A.; Guigó, R.; Martin-Subero, J.I.; Chapaprieta, V.; Beekman, R.; Martin-García, D.; Beà. S.; Salaverria, I.; Aymerich, M.; Campo, E.; Gattei, V.; Hernández, L. Expression of the transcribed ultraconserved region 70 and the related long non-coding RNA AC092652.2-202 has prognostic value in Chronic Lymphocytic Leukaemia. Br J Haematol 2018, Apr 24. DOI: 10.1111/bjh.15237.

25. Schwab, C.J.; Chilton, L.; Morrison, H.; Jones, L.; Al-Shehhi, H.; Erhorn, A.; Russell, L.J.; Moorman, A.V.; Harrison, C.J. Genes commonly deleted in childhood B-cell precursor acute lymphoblastic leukemia: association with cytogenetics and clinical features. Haematologica 2013, 98(7), 1081-1088, DOI: 10.3324/haematol.2013.085175.

26. Jones, C.L.; Bhatla, T.; Blum, R.; Wang, J.; Paugh, S.W.; Wen, X.; Teachey, D.T. Loss of TBL1XR1 disrupts glucocorticoid receptor recruitment to chromatin and results in glucocorticoid resistance in a Blymphoblastic leukemia model. J Biol Chem 2014, 289(30), 20502-20515, DOI: 10.1074/jbc.M114.569889.

27. Pang, S.H.M.; Minnich, M.; Gangatirkar, P.; Zheng, Z.; Ebert, A.; Song, G.; Huntington, N.D. PU.1 cooperates with IRF4 and IRF8 to suppress pre-B-cell leukemia. Leukemia 2016, 30(6), 1375-1387, DOI: 10.1038/leu.2016.27.

28. Shen, Y.; Park, C.S.; Suppipat, K.; Mistretta, T.A.; Puppi, M.; Horton, T.M.; Lacorazza, H.D. Inactivation of KLF4 promotes T-cell acute lymphoblastic leukemia and activates the MAP2K7 pathway. Leukemia 2017, 31(6), 1314-1324, DOI: 10.1038/leu.2016.339. 
29. Honma, R.; Goto, K.; Sakamoto, N.; Sekino, Y.; Sentani, K.; Oue, N.; Yasui, W. Expression and function of Uc. 160+, a transcribed ultraconserved region, in gastric cancer. Gastric Cancer 2017, 20(6), 960-969, DOI: 10.1007/s10120-017-0714-9.

30. Garzon, R.; Volinia, S.; Liu, C.G.; Fernandez-Cymering, C.; Palumbo, T.; Pichiorri, F.; Flomenberg, N. MicroRNA signatures associated with cytogenetics and prognosis in acute myeloid leukemia. Blood 2008, 111(6), 3183-3189, DOI: 10.1182/blood-2007-07-098749.

31. Brandalise, S.R.; Pinheiro, V.R.; Aguiar, S.S.; Matsuda, E.I.; Otubo, R.; Yunes, J.A.; Pereira, W.V.; Carvalho, E.G.; Cristofani, L.M.; Souza, M.S.; Lee, M.L.; Dobbin, J.A.; Pombo-de-Oliveira, M.S.; Lopes, L.F.; Melnikoff, K.N.; Brunetto, A.L.; Tone, L.G.; Scrideli, C.A.; Morais, V.L.; Viana, M.B. Benefits of the intermittent use of 6-mercaptopurine and methotrexate in maintenance treatment for low-risk acute lymphoblastic leukemia in children: randomized trial from the Brazilian Childhood Cooperative Group - Protocol ALL-99. J Clin Oncol, 2010, 28, 1911-1918, DOI: 10.1200/JCO.2009.25.6115.

32. FastQC: a quality control tool for high throughput sequence data, version 0.11.8; Babraham Bioinformatics, 2011

33. Ewels, P.; Magnusson, M.; Lundin, S.; Käller, M. MultiQC: summarize analysis results for multiple tools and samples in a single report. Bioinformatics, 2016, 32(19), 3047-3048, DOI: 10.1093/bioinformatics/btw354

34. Trim Galore: A wrapper tool around Cutadapt and FastQC to consistently apply quality and adapter trimming to FastQ files, version 0.5.0; Babraham Bioinformatics, 2015

35. Patro, R.; Duggal, G.; Love, M. I.; Irizarry, R. A.; Kingsford, C. Salmon provides fast and bias-aware quantification of transcript expression. Nature methods, 2017, 14(4), 417, DOI: 10.1038/nmeth.4197

36. Bray, N. L.; Pimentel, H.; Melsted, P.; Pachter, L. Near-optimal probabilistic RNA-seq quantification. Nature biotechnology, 2016, 34(5), 525, DOI: 10.1038/nbt.3519

37. Soneson, C.; Love, M. I.; \& Robinson, M. D. Differential analyses for RNA-seq: transcript-level estimates improve gene-level inferences. F1000Research, 2015, 4, DOI: 10.12688/f1000research.7563.2

38. Love, M. I.; Huber, W.; Anders, S. Moderated estimation of fold change and dispersion for RNA-seq data with DESeq2. Genome biology, 2014, 15(12), 550, DOI: 10.1186/s13059-014-0550-8 